\title{
Foundations of Unified and General Field Theories
}

\author{
L. I. Petrova
}

Faculty of Computational Mathematics and Cybernetics, Moscow State University, Russia

\begin{abstract}
The theories of skew-symmetric differential forms, which reflect the properties of conservation laws for physical fields and material systems, allow to disclose and justify the general principles of field theories. Below we present certain of concepts that lie at the basis of unified and general field theories.
\end{abstract}

Keywords Skew-Symmetric Differential Forms, Conservation Laws, Equations of Material System, Evolutionary Relation, Properties of Field Theories

\section{Introduction}

The existing field theories are based on the properties of closed exterior forms, which are invariant ones and correspond to conservation laws for physical fields. Hence, to understand the foundations of field theories and their unity, one has to know how such closed exterior forms are obtained.

In the present paper it is shown that closed exterior forms corresponding to field theories are obtained from the equations of conservation laws for energy, linear momentu $\mathrm{m}$, angular momentum, and mass, which are conservation laws for material systems (such as cosmologic systems, the systems of charged particles and so on).

The process of obtaining closed exterior forms discloses the mechanism of evolutionary processes in material systems (material media) and shows that material systems generate the physical structures, from which the physical fields are formed. This process demonstrates the connection between field theories and the equations conservation laws for material systems. Such a connection, which is common to all field theories, discloses the general foundations of field theories, their quantum character, justifies the unity of field theories and can serve as an approach to general field theory.

In present paper, beside the exterior skew-symmetric differential forms, the skew-symmetric differential forms, whose basis (in contrast to the exterior forms) are deforming manifolds, are used. Mathematical apparatus of such differential forms (which were named evolutionary ones) includes nontraditional elements like nonidentical relations and degenerate transformations and this enables one to describe evolutionary processes, discrete transitions and generation of various structures.

* Corresponding author:

ptr@cs.msu.su (L.I. Petrova)

Published online at http://journal.sapub.org/ijtmp

Copyright (C) 2012 Scientific \& Academic Publishing. All Rights Reserved

\section{Closed Inexact Exterior Forms is the Basis of Field Theories}

All existing field theories were based on the postulates of invariance or covariance, which are the closure conditions of exterior or dual form[1-4].

The properties of closed exterior and dual forms, namely, invariance, covariance, conjugacy and duality, are the basis of the group, structural and other invariant methods of field theories.

The invariant properties of closed exterior differential forms reveal the mselves explic itly or imp licitly in practically all formalis ms of field theories (the theories that describe physical fields) such as the Hamilton formalism, tensor approaches, group methods, quantum mechanics equations, the Yang-Mills theory and so on.

The gauge transformations of field theories are transformation of closed exterior forms (transformation that conserve the differential). The gauge transformations of field theories for spinor, scalar, vector and tensor fields are those of forms of $0,1,2$ and 3 degrees respectively. These are unitary transformations (0-form), gauge and canonical transformations (1-form), gradient transformations (2-form) and so on.

The gauge symmetries of field theories are those for closed exterior forms. And the interior symmetries of field theories are those of closed exterior forms, whereas the exterior symmetries of field theory equations are symmetries of dual forms.

The field theory operators are operators connected with nondegenerate trans formations of closed exterior differential forms [2].

The equations of existing field theories are those obtained on the basis of the properties of exterior form theory. Closed inexact exterior or dual forms are solutions of the field-theo ry equations. And there is the following correspondence.

-Closed exterior forms of zero degree correspond to 
quantum mechanics.

-The Hamilton forma lis $m$ bases on the properties of closed exterior and dual forms of first degree.

-The properties of closed exterior and dual forms of second degree are at the basis of the equations of electro magnetic field.

-The closure conditions of exterior and dual forms of third degree form the basis of equations for gravitational field.

One can see that both gauge transformations and field theory equations as well are connected with closed exterior forms of a certain degree. This enables one to introduce a classification of physical fields and interactions in degrees of closed exterior forms. If to denote the degree of closed exterior forms by $\mathrm{k}$, then $k=0$ corresponds to strong interaction, $k=1$ does to weak one, $k=2$ does to electromagnetic one, and $k=3$ corresponds to gravitational interaction.

Such a classification shows that there exists an internal connection between field theories that describe physical fields of various types. It is evident that the degree of closed exterior forms is a parameter that integrates field theories into unified field theory.

The connection of field theory with the theory of closed exterior forms is explained by the fact that the closure conditions of exterior and dual forms, i.e. the conditions of invariance and of covariance, correspond to conservation laws which physical fields are subject to.

As it is known, the conservation laws for physical fields are laws that point out to an existence of conserved physical quantity or object. The closed exterior form describes such conserved quantity, whereas the closed inexact exterior differential form in combination with dual form describes such a conserved object, that is, a quantity being conserved on a certain pseudostructure. The physical structures, which made up physical fields and correspond to conservation laws are just such conserved objects, namely, diffe rential-geomet rical structures made up by closed inexact exterior form and closed dual form. And the potential surfaces are those that made up by pseudostructures, which are described by closed dual forms.

One can see that the existing invariant field theories are based on the properties of closed exterior forms. A significance of exterior differential forms for field theories consists in the fact that they disclose the properties that are common for all field theories and physical fields irrespective of their specific type. This is a step to building a unified field theory.

However, the theory of closed exterior forms cannot be completed without an answer to a question of how the closed exterior forms emerge.

It will be shown below that the closed exterior forms, which describe the conservation laws for physical fields, are obtained from the skew-symmetric differential forms, which are evolutionary ones[4]. They can be derived from the equations of conservation laws for energy, linear mo mentum, angular momentum, and mass, which are conservation laws for material systems (such as thermodynamical, gas-dynami cal, cosmologic systems, the systems of charged particles and so on).

\section{Evolutionary Differential Forms as the Basis of General Field Theory}

As it was already noted, existing field theories are based on the properties of closed exterior forms, which correspond to the conservation laws for physical fields. And it will be shown that they are realized from skew-symmetric forms, which also correspond to conservation laws. Therefore we have focussed our attention on the concept of "conservation laws".

Conservation laws.

Historically it happen so that in the branches of physics connected with field theory (describing physical fields) and in physics of material systems (continuous media) the concept of "conservation laws" has a different mean ing.[The physical fields are a special form of the substance, they are carriers of various interactions such as electromagnetic, gravitational, wave, nuclear and other kinds of interactions. The material system is a variety of elements that have internal structure and interact to one another. Examples of elements that constitute the material system are electrons, protons, neutrons, atoms, fluid particles, cosmic objects and others. As examples of material systems it may be the thermodynamic, gas dynamical and cosmic systems, the systems of charged particles and others.]

In mechanics and physics of material systems (continuous media) the concept of "conservation laws" relates to the conservation laws for energy, linear momentum, angular momentum, and mass that establish the balance between the change of physical quantities and the external action. These conservation laws can be named "the balance conservation laws". The balance conservation laws are described by differential (or integral) equations $[5,6]$.

In field theory "conservation laws" are those that claim an existence of cons ervative physical quantities or objects. Such conservation laws are described by closed exterior skew-symmetric forms. The Noether theorem is an example. These are conservation laws for physical fields. (For the sake of convenience the cons ervation laws for physical fields will be called as "exact".)

It turns out that the balance and exact conservation laws are mutually connected. Closed exterior forms, which describe the conservation laws for physical fields, are obtained from evolutionary forms that in turn are derived from the equations of balance conservation laws for material systems. This follows from the analys is of the equations of balance conservation laws for material systems.

\subsection{Peculiarities of the Equations of B alance Conservation Laws for Material Systems Evolutionary Relation}

The equations of balance conservation laws (the equation $\mathrm{s}$ of mechanics and physics of material systems) is a set of 
equations that describe the conservation laws for energy, linear momentum, angular mo mentum and mass.

The functional properties and specific features of the equations of balance conservation laws depend, firstly, on a consistency of the equations made up the set of balance conservation law equations and, secondly, on the properties of conservation laws. In the analysis of these properties one obtains a relation that allows to understand the mechanism of generating closed exterior forms, which correspond to the conservation laws for physical fields and form the bas is of field theories.

\subsubsection{Research of Consistency of the Equations of Balance Conservation Law}

Let us analyze the equations that describe the conservation laws for energy and linear mo mentum.

In the accompanying reference system, which is tied to the manifold built by the trajectories of particles (elements of material system), the equation of energy is written in the form

$$
\frac{\partial \psi}{\partial \xi^{1}}=A_{1}
$$

Here $\xi^{1}$ are the coordinates along the trajectory, $\psi$ is the functional of the state that specifies material system, $A_{1}$ is the quantity that depends on specific features of the system and on external energy actions onto the system.

[The action functional, entropy, wave function can be regarded as examples of the functional $\psi$ [7].]

In a similar manner, in the accompanying reference system the equation for linear momentum appears to be reduced to the equation of the form

$$
\frac{\partial \psi}{\partial \xi^{v}}=A_{v}, \quad v=2, \ldots
$$

where $\xi^{v}$ the coordinates in the direction normal to the trajectory, $A_{v}$ are the quantities that depend on the specific features of material system and on external force actions.

Eqs. (1) and (2) can be convoluted into the relation

$$
d \psi=A_{\mu} d \xi^{\mu}, \quad \mu=1, v
$$

[Summation over repeated indices is implied.]

Relation (3) can be written as

$$
d \psi=\omega, \quad \mu=1, v
$$

Here $\omega=A_{\mu} d \xi^{\mu}$ is the skew-symmetrical differential form of the first degree.

Since the equation of the balance conservation laws are evolutionary ones, skew-symmetric differential form $\omega$ and the relation obtained (4) are also evolutionary ones.

Relation (4) has been obtained from the equation of the balance conservation laws for energy and linear momentum. In this relation the form $\omega$ is that of the first degree. If the equations of the balance conservation laws for angular momentum be added to the equations for energy and linear momentum, this form will be a form of the second degree. And, in combination with the equation of the balance conservation law for mass, this form will be a form of degree 3.

In general case the evolutionary relation can be written as

$$
d \psi=\omega^{p}
$$

where the form degree $p$ takes the values $p=0,1,2,3$. (The relation for $p=0$ is an analog to that in the differential forms, and it has been obtained from the interaction of energy and time.)

\subsubsection{The Properties of Evolutionary Relation}

Relations $(4,5)$, obtained from the equations of balance conservation laws for material systems, are describing the process of generation of closed (ine xact) exterior form. This appears to be possible due to peculiarities that this relation possesses. This relation proves to be a nonidentical self-varying relation.

Nonidentity of evolutionary relation.

To justify this we shall analyze the relation (4).

Evolutionary relation (4) is a nonidentical one as it involves the skew-symmetric differential form $\omega$, which is unclosed and cannot be a differential like the left-hand side of this relation. The evolutionary form $\omega$ is not closed since the differential of evolutionary form $\omega$ and its commutator are nonzero. The differential of evolutionary form $\omega$ is expressed as $d \omega=K_{1 v} d \xi^{1} d \xi^{v}$, where $K_{1 v}$ are components of the form commutator. Without accounting for terms that are connected with the deformation of the manifold made up by the trajectories, the components of the commutator of such a form can be written as follows:

$$
K_{1 v}=\left(\frac{\partial A_{\beta}}{\partial \xi^{\alpha}}-\frac{\partial A_{\alpha}}{\partial \xi^{\beta}}\right)
$$

The coefficients $A_{\mu}$ of the form $\omega$ have been obtained ether from the equation of the balance conservation law for energy or from that for linear momentum. This means that in the first case the coefficients depend on the energetic action and in the second case they depend on the force action. In actual processes the energetic and force actions have different nature and appear to be inconsistent. The commutator of the form $\omega$ constructed of the derivatives of such coefficients is nonzero. This means that the differential of the form $\omega$ is nonzero as well. Thus, the form $\omega$ proves to be unclosed and cannot be a differential. In the left-hand side of relation (4) it stands a differential, whereas in the right-hand side it stands an unclosed form that is not a differential. Such a relation cannot be an identical one. This is also true for relation (5).

Nonidentity of the evolutionary relation obtained from the equations of conservation laws material systems means that the balance conservation law equations are inconsistent. And this points out to the fact that the balance conservation laws are noncommutative[8]. The equations of balancenoncomm 
utative conservation laws describe not only the state of material systems. They control evolutionary processes in material systems that are accompanied by an emergence of physical structures, made up physical fields.

Self-variation of the evolutionary nonidentical relation.

The evolutionary nonidentical relation is self-varying, because, firstly, it is a nonidentical, namely, it contains two terms one of which appears to be unmeasurable, and, secondly, it is an evolutionary relation, that is, the variation of any term of the relation in some process leads to a variation of another term; and, in turn, the variation of the latter leads to variation of the former. Since one of the terms is an unmeasurable quantity, the other cannot be compared with the first one, and hence, the process of mutual variation cannot stop.

Nonidentical self-varying evolutionary relation obtaind fro $m$ the equations of conservation laws for material systems is a relation for a state functional. Be low it will be shown that this relation, firstly, has a mathematical meaning, namely, it allows to disclose the mechanis $m$ of generation of closed exterior forms, which correspond to the conservation laws for physical fields, and points out to a rise of physical structures and, secondly, has a physical meaning, namely, it describes a physical structure generation and formatting physical fields. This allows to understand what lies at the basis of field theories.

\subsection{Mechanism of Obtaining Closed Exterior For ms Degenerate Transfor mation}

As it was already noted, existing field theories are based on the properties of closed exterior forms, which correspond to the conservation laws for physical fields. And it will be shown that they are realized from the evolutionary skew-symmetric form, which enters into the evolutionary relation obtained from the equations of conservation laws for material system.

Closed exterior forms are obtained from evolutionary forms only as a result of a certain evolutionary process. This is due to the fact that the evolutionary form is unclosed, namely, the differential of this form is nonzero whereas the differential of closed exterior form vanishes. Hence, a closed exterior form can be obtained from evolutionary form only under degenerate transformation, i.e. under a transformation that does not conserve a differential. Th is transformation (the tangent transformation is an example) is realized only under additional conditions. Such additional conditions may be realized spontaneously while self-vary ing the nonidentical relation.

If the conditions of degenerate transformation are rea lized, from the unclosed evolutionary form with nonvanishing differential $d \omega^{p} \neq 0$, one can obtain a differential form closed on pseudostructure. The differential of this form equals zero. That is, it is realized the transition

$$
d \omega^{p} \neq 0 \rightarrow d_{\pi} \omega^{p}=0, d_{\pi}^{*} \omega^{p}=0
$$

The condition

$$
d_{\pi}^{*} \omega^{p}=0
$$

is an equation of a certain pseudostructure $\pi$ on which the differential of evolutionary form vanishes:

$$
d_{\pi} \omega^{p}=0
$$

That is, the closed (inexact) exterior form $\omega_{\pi}^{p}$ is obtained on pseudostructure.

Thus, if the conditions for a degenerate transformation are realized, from an unclosed evolutionary form can obtain a differential form closed on a pseudostructure, that is closed inexact exterior form. Such closed inexact exterior form corresponds to conservation laws for physical fields. As it was noted earlier, closed and dual forms produce differential-geometrical structure (a pseudostructure with a conservative quantity), which corresponds to the conservation law.

The physical structures that form physical fields are just such differential-geometric structures, which correspond to the conservation laws for physical fields (conservative quantities or objects).

On pseudostructure $\pi$ realized, from evolutionary nonidentical relation (5), it follows the relation

$$
d_{\pi} \psi=\omega_{\pi}^{p}
$$

which appears to be an identical relation. Since the form $\omega_{\pi}^{p}$ is a closed one, on the pseudostructure this form turns out to be a differential. There are differentials in the left-hand and right-hand sides of this relation. This means that the relation obtained is an identical one. As it will be shown below, such identical relation has unique physical mean ing.[Pseudostructures are obtained from the condition of degenerate transformation of the evolutionary relation. Conditions of degenerate transformation are realized at vanishing some functional expressions such as Jacobians, determinants, the Poisson brackets, residues, and others. They are connected with the symmetries, which can be due to the degrees of freedom of the material system (for example, the translational, rotational and oscillatory freedom of the material system). Pseudostructures constitute the integral surfaces such as characteristics, singular points, potentials of simple and double layers and others.

Mathematically, a degenerate transformation is realized as a transition (nonequivalent) from the noninertial frame of reference to the loccally-inertial frame of reference. Specially, at describing the material system this is a transition from accompanying (noninertial) frame of reference to pseudostructures. The evolutionary form and nonidentical evolutionary relation are defined in the noninertial frame of reference (on nonintegrable manifold). But the thereby obtained closed exterior forms are obtained with respect to the locally-inertial frame of reference (on integrable pseudostructure).]

It has been shown that from the equations of conservation laws for material systems it is obtained the evolutionary form, which generates closed inexact exterior forms, which 
correspond to conservation laws for physical fields and describes physical structures.

[The identical relation can be directly integrated because it involves closed forms that are differentials. In the integration process a transition to the relation with differential forms of lesser degrees takes place. An integration and transitions with lowering the form degree are allowed in nonidentical relations as well but only in the case of degenerate transformations. By integrating (under realization of re levant degenerate trans form) the nonidentical evolutionary relation with the forms of degree $p$, one can successively obtain identical relations with the forms of degree $k$, where $k$ takes the values $p, p-1, \ldots, 0$. At each transition the closed forms on the pseudostructure of sequential degrees $k=p, k=p-1, \ldots, k=0$, are formed, which indicates the creation of physical structures.]

Below it will be shown that the nonidentical evolutionary relation also describes a generation of physical structures.

\subsection{Mechanism of Evolutionary Processes in Material Systems Origination of Physical Structures}

The evolutionary relation includes the differential $d \psi$ that specifies the state of material system. Since the relation is nonidentical (this is due to noncommutativity of the conservation laws), one cannot obtain the differential from this relation. This points to the fact of absence of the state function and the nonequilibrium state of the material system.

The nonequilibrium state means that there is an internal force in the material system. It is evident that the internal force originates at the expense of some quantity described by the evolutionary form commutator. (If the balance conservation laws be commutative, the evolutionary form commutator be zero, and this would point to the equilibrium state, i.e. the absence of internal forces.) Everything that give contribution to the evolutionary form commutator leads to emergence of internal force.

Evolutionary relation also describes a variation of nonequilibrium state. Self-variation of the nonidentical evolutionary relation points to the fact that the state of material system turns out to be self-varying. It is evident that this self-variation proceeds under the action of internal force whose quantity is described by the commutator of unclosed evolutionary form $\omega^{p}$. In this process the state of material system changes but remains nonequilibrium because the internal forces do not vanish due to that the evolutionary form commutator remains to be nonzero.

The transition from nonidentical relation to identical one points to the transition of material system from nonequilib ri um state to locally-equilib rium state. From identical relation one can obtain the differential of state. This points to availability of the state function and the transition of material system to locally equilibrium state (such a transition is a local one since the identical relation is realized only on a certain structure).

The transition from nonequilib rium state to locally-equili brium one means that the material system has been locally got rid of internal force. This follows from the evolutionary relation. As it has been shown, the transition from nonidentical relation to identical one is realized under degenerate transformation when it is proceeded a transition from evolutionary form with the commutator (which describes internal forces) being nonzero to the closed exterior form with vanishing commutator, and this points out to an absence of internal force.

Realization of the conditions of degenerate trans formation, which describes a transition of material system from nonequilibrium state to locally-equilibrium one, are conditioned by the degrees of freedom of material system. The presence of the degree of freedom allows the inconsistent quantities to redistribute and transform to measurable quantities of the system itself. The measurable quantities of material system realized reveal in material system as the spontaneous emegence of observable measurable formations. Waves, vortices, fluctuations, turbulent pulsations and so on are examples of such formations.

One can see that the transition of material system from nonequilibrium state the locally-equilibrium one is accompanied by the emergence of observable formations in material system.

On the other hand, as it has been shown, the transition from nonidentical relation to identical one, which describes the transition of material system from nonequilibrium state the locally-equilibrium one, occurs at realization closed inexact exterior and dual forms. This points out to a realization of a differential-geo metrical structure, namely, a pseudostructure with conservative quantity, which corresponds to the conservation law. As it was already noted, the physical structures that form physical fields are just such differential-geometric structures, which correspond to the conservation laws for physical fields (conservative quantities or objects). (Massless particles, structures made up by eikonal surfaces and wave fronts, and so on are examples of physical structures.)

[The characteristics of physical structure and formations are determined by the evolutionary form, the evolutionary form commutator, and additional conditions, which are specified by the degenerate transformation. The connection of physical structures with skew-symmetric differential forms allows to introduce a classification of these structures in dependence on parameters that specify skew-symmetric differential forms (degree of evolutionary form $p$ and degree of closed exterior form $k$. A type of physical structure (and of physical fields made up by these structures) depends on the degree of closed exterior form realized, which corresponds to a number of conservation law equations that appear to be consistent. This is, a type of physical structure depends on a number of balance conservation laws that turn out to be commutative ones (as a result of evolutionary process).]

Thus can see, that the transition of material system from nonequilibrium state the locally-equilibrium one is accompanied by orig ination of physical structures and the 
emergence of observable formations in material system.

Physical structures and observed formations are a man ifestation of the same phenomenon. (Light is an example of manifestation of such a duality, namely, as a massless particle (photon) and as a wave.) However, physical structures and observed formations are not identical objects. Whereas the wave is an observable formation, to the physical structure it is assigned the eikonal (that is, the moving wave element made up a physical structure).

Duality of physical structures and observed formations is described by identical relation (6). The left-hand side of this relation includes the differential, which specifies material system and whose availability points to the locally-equilibri um state of material system and an emergence of measurable formations. And the right-hand side includes a closed ine xact form, which is a characteristic of physical structures.

It is evident that the identical relation obtained from nonidentical evolutionary relation has an unique meaning. This relation discloses a connection between physical fields and material systems.

[It should emphasize once more that the connection of physical fields with material systems is realized by the conservation laws. The closed inexact exterior forms, which describe the conservation laws for physical fields, are obtained from the evolutionary forms, which enter into nonidentical relation derived from the equations of noncommutative conservation laws for material systems. It is just the noncommutativity of balance conservation laws plays a governing role in evolutionary processes, which proceed in material media and lead to generating physical fields.]

It was shown that the physical structures, of which physical fields are made up, are generated by material systems.

This means that there exists a connection between the field theory equations and the equations for material systems. And this connection is realized with the help of nonidentical evolutionary relation.

\subsection{Specific Features of the Field-The ory Equations}

The field-theory equations are equations for physical fields. As the physical fields are described by the closed exterior (inexact) forms, it is obvious that solutions to the field-theory equations must be closed exterior forms, i.e. to be differentials. And such differentials, which are closed exterior forms, can be obtained from the nonidentical evolutionary relation.

One can see that all field-theory equations have the form of relations. They can be relations in differential forms or in the forms of their tensor or differential (i.e. expressed in terms of derivatives) analogs.

The Einstein equation is a relation in differential forms. This equation relates the differential of the first degree form (Einstein's tensor) and the differential form of second degree, namely, the energy-momentum tensor. (It should be noted that Einstein's equation follows from the differential form of third degree).

The Dirac equation relates Dirac's bra- and cket- vectors, which made up the differential form of zero degree.

The Maxwell equations have the form of tensor relations.

The Schrodinger's equations have the form of relations expressed in terms of derivatives and their analogs.

From the field-theory equations, as well as from the nonidentical evolutionary relation, the identical relation, which contains the closed exterior form, is obtained.

The closed exterior forms or their tensor or differential analogs, which are obtained from identical relations, are solutions to the field-theory equations.

As one can see, from the field-theory equations it follows such identical relation as

1) the Dirac relations made up of Dirac's bra- and cketvectors, which connect a closed exterior form of zero degree;

2) the Poincare invariant, which connects closed exterior forms of first degree;

3) the relations

$$
d \theta^{2}=0, d^{*} \theta^{2}=0
$$

are those for closed exterior forms of second degree obtained from Maxwell equations;

4) the Bianchi identity for gravitational field.

From the Einstein equation it is obtained the identical relation in the case when the covariant derivative of the energy-mo mentum tens or vanishes.

It is evident that all equations of existing field theories are in essence the relations that connect skew-symmetric forms or their analogs.

It may be emphasized once mo re that the equations of field theories have the form of relations for functionals [7] such as wave function (the relation corresponding to differential form of zero degree), action functional (the relation corresponding to differential form of first degree), the Pointing vector (the relation corresponding to differential form of second degree). The tensor functionals that correspond to Einstein's equation are obtained from the relation connecting the differential forms of third degree.

The nonidentical evolutionary relations derived from the equations for material media, as it was already mentioned, unites the relations for all these functionals. That is, all equations of field theories are analogous to the nonidentical evolutionary relation.

From this it follows that the nonidentical evolutionary relation, obtained from the equations of conservation laws for material system, can play a role of the equation of general field theory that discloses common properties and peculiarities of existing equations of field theory.

This fact points out to a connection between the field-theory equations and the equations of conservation laws for material systems.

It is necessary to emphasize that the evolutionary relation which is obtained from the equations of conservation laws for material systems, is the relation for functionals such as wave function, functional action, entropy and others, in other words, for functional of field theories, which are based on 
properties of conservation laws for physical fields. And this points to the fact that conservation laws for physical fields are connected with $\mathrm{c}$ conservation laws for material systems.

\subsection{General Properties of Physical Fields and Field The ories}

\section{One can Underline the Following Results}

1. The existing invariant field theories are based on the properties of closed exterior forms, which correspond to conservation laws for physical fields.

The degree of closed exterior forms is a parameter that integrates field theories into unified field theory.

2. The closed exterior and dual forms, on which properties field theories are based, are realized from the evolutionary forms obtained from the equations of conservation laws for material systems, namely, the conservation laws of energy, linear momentum, angular mo mentum, and mass.

3. The internal and external symmetries of field theories, which are those of closed exterior and dual forms, are conditioned by the degrees of freedo m of material media.

The symmetries of field theories are those of exterior and dual forms, namely, of the closure conditions for exterior and dual forms. So, the gauge symmetries, which are interior symmetries of field theories, are symmetries of closed exterior forms. And the exterior symmetries of field theory equations are those of relevant dual forms.

The interior symmetries of field theories relate to the conservation laws for physical fields, whereas the exterior symmetries of field theories, which are conditioned by the degrees of freedom of material systems, relate to the equations of the balance conservation laws for material systems.

4. The nondegenerate transformations of exterior differential forms are connected with interior symmetries of field theories, and the degenerate transformations of evolutionary forms are connected with exterior symmetries of field theories.

Nondegenerate transformations of field theories (the transformations of closed exterior forms, transformations that conserve the differential) relate to the degenerate transformations of evolutionary forms obtained from the equations for material systems.

[The degenerate transformations is a transition from evolutionary forms to closed exterior form. And the nondegenerate transformation is a transition from any closed form to another closed form. Since the closed exterior form and the closed dual form made up the physical structure, the degenerate transformation is an emergence of physical structure, whereas the nondegenerate transformation is a transition from any physical structure to another one.]

5. The physical structures, which constitute physical fields, are generated by material systems.

The origination of physical structures, from which physical fields are made up, proceeds discretely under realization of the degrees of freedom of material systems. This explains the quantum character of field theories.
6. The parameter which unites field theories is a degree $k$ of the closed exterior form being realized. This parameter is connected with a number of balance conservation laws, which become commuative ones as a result of evolutionary process.

7. The constants and characteristics of field theories are connected with characteristics of relevant material systems. (But this connection is indirect. This connection is realized in evolutionary process).

8. The field theories are connected with the equations of noncommutative conservation laws for material media (the balance conservation laws of energy, linear momentum, angular mo mentum, and mass).

\section{Conclusions}

The fundamental result that clarifies the problems of field theories is the fact that the field theories, which are based on the conservation laws for physical fields, are connected with the equations of noncommutative conservation laws for material systems (the conservation laws of energy, linear momentum, angular momentum, and mass as well as the analog of such laws for the time, which takes into account the noncommutativity of time and energy of material system).

Such a connection, which is common to all field theories, discloses the general foundations of field theories. This connection has to be taken into account while building the general field theory.

It should be once more pointed out to unique role of skew-symmetric differential forms for physical theory. Closed exterior forms describe the properties that are common for all field theories. This discloses an internal connection between existing field theories and can be considered as an approach to build ing an unified field theory. And the evolutionary forms, which generate closed exterior forms, disclose the basis on which field theories are built (a connection of field-theory equations with the equations for material systems), and this can disclose a meaning of postulates forming the basis of field theories. The theory of skew-symmetric differential forms, which discloses a connection between existing field theories and their basis, can appear to be helpful in solving the problems of existing field theories and building a general field theory.

\section{REFERENCES}

[1] Cartan E., Les Systemes Differentials Exterieus ef Leurs Application Geometriques. -Paris, Hermann, 1945.

[2] Wheeler J.A., Neutrino, Gravitation and Geometry. Bologna, 1960.

[3] Weinberg S., Gravitation and Cosmology. Principles and applications of the general theory of relativity. Wiley \& Sons, Inc., N-Y, 1972. 
[4] Petrova L.I. Exterior and evolutionary differential forms in mathematical physics: Theory and Applications. -Lulu.com, 2008, 157pp.

[5] Tolman R.C. Relativity, Thermodynamics, and Cosmology. Clarendon Press, Oxford, UK, 1969.

[6] Clarke J.F., Machesney M. The Dynamics of Real Gases. Butterworths, London, 1964.
[7] Petrova L.I. Physical meaning and a duality of concepts of wave functional, entropy, the Pointing vector, the Einstein tensor. Journal of Mathematics Research, Vol. 4, No. 3, 2012.

[8] Petrova L.I. The noncommutativity of the conservation laws: Mechanism of origination of vorticity and turbulence. International Journal of Theoretical and Mathematical Physics, Vol.2, No.4, 2012.. 